\title{
Consumo de tabaco en adolescentes y jóvenes de México que no estudian y no trabajan
}

\author{
Marycarmen Bustos Gamiño', Jorge A. Villatoro Velázquez', Natania Oliva Robles', Miguel Ángel López Brambila², \\ Diana Anahí Fregoso Ito', María Elena Medina-Mora' \\ ' Instituto Nacional de Psiquiatría Ramón de la Fuente Muñiz, México \\ ${ }^{2}$ Instituto para la Atención y Prevención de las Adicciones en la Ciudad de México, México
}

\section{RESUMEN}

Introducción: la falta de oportunidades de estudio o trabajo incrementa la vulnerabilidad de los jóvenes, quienes ante la inestabilidad pueden iniciarse en el consumo de tabaco o de otras sustancias adictivas. Objetivo: analizar cómo es el consumo de tabaco entre grupos de adolescentes y jóvenes que sólo estudian, sólo trabajan, estudian y trabajan, o no estudian ni trabajan. Método: se tomó una muestra nacional de 6 mil 72 jóvenes de 12 a 25 años con datos que provienen de la Encuesta Nacional de Adicciones 2011, con un diseño aleatorio, probabilístico y polietápico. Se midió el consumo alguna vez, en el último año y en el último mes, así como el uso diario de tabaco y el consumo de más de 100 cigarros. Resultados: los hombres de 12 a 17 años que no estudian y no trabajan tienen un consumo de tabaco mayor que aquellos que sólo estudian. Tanto en hombres como en mujeres de 12 a 25 años, quienes trabajan presentan un consumo mayor de tabaco que los jóvenes que sólo estudian. Discusión y conclusiones: el riesgo de consumo de sustancias no se da únicamente entre los jóvenes que no estudian y no trabajan, sino también en aquellos que estando en edad de estudiar, se encuentran laborando, por lo que es importante reforzar y actualizar los programas de prevención del consumo de tabaco que han permitido contener, y en algunos casos disminuir, los niveles de consumo de la población.

Palabras clave: tabaco; empleo; estudio; jóvenes; salud mental; consumo de sustancias.

\begin{abstract}
Introduction: the lack of opportunities to study or work increases the vulnerability of adolescents, who given this situation may start the consumption of tobacco or other addictive substances. Objective: the aim of this study is to analyze tobacco consumption patterns among four youth groups: those who only study, only work, study and work and who don't study and don't work. Method: a national sample of 6, 072 adolescents from 12 to 25 years with data from the National Survey of Addictions 2011, carried out with a random, probabilistic and multistage design. Consumption was measured lifetime, last year, last month, daily use and consumption of more 100 or more cigarettes. Results: men aged 12 to 17 who do not study and do not work have a higher consumption rate than those who only study; men and women aged 12 to 25 years who work have higher consumption rates compared to young people who only study. Discussion and conclusions: the risk of using substances does not occur only on young people who do not study and do not work, but also on those that being school-aged are currently working, so it is important to strengthen and update tobacco consumption prevention programs that have helped to contain, and in some cases decline the consumption rates among the population.
\end{abstract}

Key words: tobacco; employment; schooling; adolescents; mental health; substance use.

\footnotetext{
Autor de correspondencia:

Jorge A. Villatoro Velázquez. Instituto Nacional de Psiquiatría Ramón de la Fuente Muñiz. Calzada México Xochimilco núm. 101, col. San Lorenzo Huipulco, del. Tlalpan, C.P. 14370, Distrito Federal, México. Correo electrónico: ameth@imp.edu.mx.

Recibido: 01 de agosto del 2015.

Aceptado: 18 de septiembre del 2015.

DOI: 10.28931/riiad.2015.1.05
} 


\section{INTRODUCCIÓN}

Actualmente los jóvenes se enfrentan a dificultades como la falta de acceso a la educación y al empleo, debido a causas económicas, sociales o culturales. Estas situaciones pueden generar problemas de salud mental, pérdida de identidad y sensación de incertidumbre hacia el futuro (Székely, 2011). El fenómeno de la inactividad en los jóvenes afecta a muchos países; en Latinoamérica, cerca de 16 millones de personas (25\%) de entre 15 y 25 años de edad se encuentran en esta situación (Székely, 2011). Los países que resultan mayormente afectados son Honduras, Nicaragua, Guatemala y El Salvador (Székely, 2011; D’Alesandre, 2010). En México, cerca de 23\% de las personas de 12 a 29 años se encuentra en este estatus (Instituto Nacional de Estadística y Geografía [INEGI], 2012).

La falta de actividades como el estudio o el trabajo incrementa la vulnerabilidad de los jóvenes y puede generar disminución de la autoestima, depresión, ansiedad y un sentimiento de insatisfacción, que aunados a otros factores, pueden llevarlos al consumo de sustancias y a otras conductas problemáticas (Organización Panamericana de la Salud [OPS], \& Instituto Nacional de Salud Pública [INSP], 2010; Benjet et al., 2009; Pérez et al., 2010; Salazar, Torres, Reynaldos, Figueroa, \& Araiza, 2011; Benjet et al., 2012; Gutiérrez, Martínez, Pacheco, \& Benjet, 2014).

En este sentido, la literatura señala que una de las drogas de inicio más comunes es el tabaco; el inicio en el consumo de esta sustancia se reporta principalmente durante la adolescencia (Organization of American States [OAS] \& Inter-American Drug Abuse Control Commission [CICAD], 2010; Villatoro et al., 2010; Reynales et al., 2011; Johnston, O’Malley, Miech, Bachman, \& Schulenberg, 2015; Organización de Estados Americanos \& CICAD, 2015), lo que incrementa el riesgo de desarrollar dependencia de la nicotina (Anderson, 2006; Reynales et al., 2012) y de involucrarse en el uso de otras sustancias adictivas (Villalobos \& Rojas, 2007; Reynales-Shigematsu et al., 2011; Villatoro et al., 2014; Organización de los Estados Americanos [OEA] \& Comisión Interamericana para el Control del Abuso de Drogas [CICAD], 2015).

Al respecto, en los países del Caribe, la edad promedio de inicio del consumo de tabaco en los adolescentes es de 11.9 años (OAS \& CICAD, 2010); en México, la edad de inicio promedio es de 13.7 años. De acuerdo con la Encuesta Nacional de Adicciones 2011, 21.7\% de la población de 12 a 65 años es fumadora activa; y $21.5 \%$ tiene entre 12 y 25 años de edad (Reynales et al., 2012).

Se ha señalado que dedicarse únicamente a la escuela suele ser un factor protector ante el consumo de tabaco. Las investigaciones indican que quienes sólo estudian reportan una menor prevalencia de consumo, que aquellos que además de estudiar trabajan o que aquellos que ya no estudian (Nuño-Gutiérrez, Álvarez-Nemegyei, Madrigal-De León, \& Rasmussen, 2005; Reddy-Javobs, Téllez-Rojo, Meneses-González, Campuzano-Rincón, \& Hernández-Ávila, 2006; National Center on Addiction and Substance Abuse [CASA] \& Califano, 2007; Nuño-Gutiérrez, Álvarez-Nemegyei, Madrigal-De León, \& Tapia-Curiel, 2008; Welte, Barnes, Tidwell, \& Hoffman, 2011; Villatoro et al., 2014). Una investigación llevada a cabo con población mexicana indicó que la prevalencia de consumo de 100 cigarrillos o más fue de $4.7 \%$ en los adolescentes que mencionaron asistir a la escuela, lo que contrasta con el $17.2 \%$ de los que reportaron no asistir (Villalobos \& Rojas, 2007).

Si bien México cuenta con un amplio número de investigaciones acerca del consumo de tabaco en relación con distintos factores, pocos son los trabajos que han abordado, en encuestas nacionales, el uso de esta sustancia en adolescentes y jóvenes que no estudian y no trabajan (Benjet et al., 2012).

En este contexto, el objetivo de este estudio es conocer la prevalencia de consumo de tabaco en relación al estatus laboral y escolar en mexicanos de 12 a 25 años.

\section{MÉTODO}

\section{Diseño}

Este trabajo representa un análisis secundario de la Encuesta Nacional de Adicciones 2011 realizada en México. La descripción metodológica fue publicada y puede consultarse en Reynales et al. (2012). Dicho estudio fue una encuesta realizada en hogares, con representatividad nacional y para ocho regiones del país, tanto en zonas rurales como urbanas. El diseño muestral fue aleatorio, probabilístico y polietápico; empleó los registros del Censo de Población y Vivienda del 2010 para obtener la muestra. El cuestionario aplicado fue computarizado. En cada hogar se seleccionó a un adulto de entre 18 y 65 años, y a un adolescente de entre 12 y 17 años, siempre y cuando la composición del hogar lo permitiera. La selección de éstos se llevó a cabo mediante un muestreo aleatorio simple en cada uno de los dos grupos de edad. El tamaño final de la muestra fue de 16 mil 249 entrevistas completas, con una tasa de respuesta global de $73.3 \%$.

El cuestionario individual estuvo compuesto por distintas secciones tales como datos sociodemográficos, consumo, exposición, percepción y tratamiento de sustancias adictivas como tabaco, alcohol, drogas médicas e ilegales, entre otras. 


\section{Consideraciones éticas}

El estudio fue aprobado por los Comités de Ética del INSP y el Instituto Nacional de Psiquiatría. Todos los participantes otorgaron consentimiento informado previo a la recolección de datos. En el caso de los menores de edad se recabó, además de su consentimiento, la autorización de los padres.

\section{Participantes}

Dentro del hogar se seleccionó a un integrante adulto de entre 18 y 65 años y a un integrante adolescente de 12 a 17 años, si las características del hogar lo permitían, mediante una selección aleatoria simple. Para efectos de este trabajo, se consideró únicamente a la población de 12 a 25 años que participó en el estudio, lo que representa 37.4\% (6 mil 72 jóvenes) de la muestra total; 3 mil 57 de los cuales fueron hombres y 3 mil 15, mujeres.

\section{Procedimiento}

Grupos de edad. Se formaron dos grupos de edad para los análisis de consumo de tabaco y el estatus escolar-laboral. El primer grupo, integrado con jóvenes de 12 a 17 años, corresponde al de los menores de edad (2 mil 742 en total, mil 389 hombres y mil 353 mujeres); en tanto que el segundo, con jóvenes de 18 a 25 años (3 mil 330 en total, mil 668 hombres y mil 662 mujeres), contempla a personas que, si bien ya son mayores de edad, pueden estar estudiando aún.

Consumo de tabaco. Para los indicadores de consumo de tabaco, se incluyeron aquellos considerados internacionalmente por la OMS (Department of Health and Human Services, Center for Disease Control and Prevention [CDC] \& Organización Mundial de la Salud [OMS], 2011).

- Consumo alguna vez: se refiere a la prevalencia global de consumo en la vida, aun cuando el individuo haya usado productos de tabaco una sola vez. La prevalencia como tal es el porcentaje de individuos que reúnen esta condición, entre el total de individuos del mismo grupo de referencia.

- Consumo último año: se refiere al uso de productos de tabaco en los doce meses previos al levantamiento del estudio; a los fumadores que consumen en el período descrito se les menciona como fumadores activos. La prevalencia como tal es el porcentaje de individuos que reúnen esta condición, entre el total de individuos del mismo grupo de referencia.

- Consumo último mes: se refiere al consumo de productos de tabaco en los treinta días previos a la realización del estudio; también se le conoce como preva- lencia actual. La prevalencia como tal es el porcentaje de individuos que reúnen esta condición, entre el total de individuos del mismo grupo de referencia.

- Consumo de 100 cigarros o más: se refiere a haber fumado esta cantidad de cigarros o bien cinco cajetillas, a lo largo de la vida.

- Consumo diario: patrón de consumo que se caracteriza por fumar uno o más cigarros por día.

Estatus escolar-laboral. Compuesta de los valores sólo estudiar, sólo trabajar, estudiar y trabajar, y no estudiar y no trabajar. Se consideró "estudiar" cuando, al momento de la entrevista, el joven indicó estar inscrito en una institución educativa y asistir a clases. Para "trabajar", se consideró a la población que mencionó haber laborado en los últimos 30 días previos a la encuesta.

\section{Análisis estadísticos}

Los análisis descriptivos se obtuvieron mediante el programa SPSS versión 21, mientras que las estimaciones de los intervalos de confianza se realizaron mediante el programa STATA versión 13. Su estimación se realizó considerando el diseño del estudio para ajustar el tamaño de los errores estándar.

\section{RESULTADOS}

En la Tabla 1 se presenta la proporción de hombres y mujeres de 12 a 17 años y de 18 a 25 años, según su estatus escolar o laboral. Más de la mitad de los entrevistados de 12 a 17 años estudian, y un porcentaje bajo (4.7\%) no estudia y no trabaja. Por el contrario, la mayor parte de quienes tienen 18 a 25 años tienen un trabajo y aquellos sin ninguna actividad representan el 9.3\%.

Se obtuvieron los intervalos de confianza de cada indicador para determinar si existen diferencias significativas en el consumo de los diferentes grupos.

Los resultados estadísticos de cada uno de los indicadores de consumo de tabaco se muestran en la Tabla 2.

\section{Consumo alguna vez}

En los hombres de 12 a 17 años se encontró un consumo mayor en los adolescentes que trabajan (49.3\%), comparados con los que estudian. En hombres de 18 a 25 años, quienes estudian (43.4\%) tienen un consumo menor en comparación con los otros tres grupos. Cuando se analiza el uso de la sustancia entre grupos de edad, se observa un consumo superior en el grupo de 18 a 25 años en cada una de las categorías. 
Tabla 1

Población de 12 a 25 años según sexo y ocupación

\begin{tabular}{|c|c|c|c|c|c|c|c|c|}
\hline & \multicolumn{2}{|c|}{ Estudia } & \multicolumn{2}{|c|}{ Trabaja } & \multicolumn{2}{|c|}{ Estudia y trabaja } & \multicolumn{2}{|c|}{ No estudia y no trabaja } \\
\hline & $\mathrm{N}$ & $\%$ & $\mathrm{~N}$ & $\%$ & $\mathrm{~N}$ & $\%$ & $\mathrm{~N}$ & $\%$ \\
\hline \multicolumn{9}{|l|}{ Hombres } \\
\hline 12 a 17 & 4685152 & 68.8 & 959690 & 14.1 & 871403 & 12.8 & 291233 & 4.3 \\
\hline 18 a 25 & 1636018 & 20.0 & 4743859 & 58.0 & 1069967 & 13.1 & 723995 & 8.9 \\
\hline \multicolumn{9}{|l|}{ Mujeres } \\
\hline 12 a 17 & 5045625 & 76.1 & 969116 & 14.6 & 279334 & 4.2 & 336022 & 5.1 \\
\hline 18 a 25 & 1582376 & 19.4 & 5399815 & 66.3 & 370254 & 4.5 & 790415 & 9.7 \\
\hline \multicolumn{9}{|l|}{ Total } \\
\hline 12 a 17 & 9730777 & 72.4 & 1928806 & 14.4 & 1150736 & 8.6 & 627255 & 4.7 \\
\hline 18 a 25 & 3218394 & 19.7 & 10143675 & 62.2 & 1440221 & 8.8 & 1514411 & 9.3 \\
\hline
\end{tabular}

En las mujeres de 12 a 17 años existe un menor consumo en aquellas que estudian (13.3\%), en comparación con las que trabajan o estudian y trabajan. En el grupo de 18 a 25 años de edad no se observan diferencias en el consumo entre las distintas categorías. Por grupos de edad, existe un mayor consumo en las jóvenes de 18 a 25 años que estudian (44.6\%) o trabajan (39.3\%), en comparación con las de 12 a 17 años.

Por sexo, los hombres de 12 a 17 años de edad que estudian $(21.6 \%)$ o que trabajan (49.3\%) tienen un consumo mayor al de las mujeres de la misma edad, mientras que el uso de la sustancia es similar en ambos sexos para el resto de las categorías. Por su parte, en el grupo de 18 a 25 años, los hombres presentan un consumo mayor que el de las mujeres de esta misma edad en las categorías de los que trabajan (71.3\%) y de quienes no estudian y no trabajan $(73.1 \%)$.

\section{Consumo último año}

Los hombres de 12 a 17 años que estudian (11.3\%) tienen un consumo menor que aquellos que trabajan o que no estudian ni trabajan; también, quienes trabajan (36.0\%) presentan un uso de tabaco mayor que los que estudian y trabajan. Del grupo de hombres con edades de 18 a 25 años, quienes trabajan (47.3\%) presentan un consumo más alto en comparación con los que únicamente estudian. Por grupos de edad, sólo en la categoría de los que estudian, se encuentra un uso mayor en los de 18 a 25 años (25.7\%) que en los adolescentes de 12 a 17 años.

Para las mujeres con edades de 12 a 17 años, así como de 18 a 25 años, el consumo es similar entre las diferentes categorías. Aquellas jóvenes de 18 a 25 años que estudian (22.7\%) presentan un consumo mayor al de las adolescentes de 12 a 17 años. En las otras tres categorías, las prevalencias son similares en ambos grupos de edad.
Al analizar los datos entre ambos sexos, se encontró que los hombres de 12 a 17 años que estudian (11.3\%) o trabajan (36.0\%) tienen un consumo más alto que las mujeres de las mismas categorías. En el rango de 18 a 25 años, los hombres que trabajan (47.3\%) o que no estudian ni trabajan (38.6\%) presentan un consumo mayor que las mujeres de estas mismas categorías.

\section{Consumo último mes}

En este indicador de consumo, los hombres de 12 a 17 años que trabajan (29.4\%) tienen un consumo más alto que aquellos que sólo estudian o que estudian y trabajan. Además, quienes no estudian y no trabajan (18.3\%) presentan un consumo superior a los que estudian. En el rango de 18 a 25 años, los que estudian (15.6\%) reportan un consumo de tabaco menor que quienes trabajan. Por grupos de edad, el consumo entre los adolescentes de 12 a 17 años y el de los jóvenes de 18 a 25 es similar en las diferentes categorías.

En las mujeres, las adolescentes de 12 a 17 años que sólo trabajan (8.0\%) tienen un consumo de tabaco mayor que quienes sólo estudian. En el rango de 18 a 25 años, el consumo es similar entre las diferentes categorías. Por grupos de edad, únicamente se observan diferencias en la categoría de mujeres que estudian, en la cual quienes se hallan entre los 18 y 25 años (14.7\%) presentan un consumo superior a las de menor edad.

Por sexo, en el grupo de 12 a 17 años, el consumo de tabaco es más alto en los hombres que estudian (6.4\%), en los que trabajan (29.4\%) y en quienes no estudian y no trabajan (18.3\%), en comparación con las mujeres de estas mismas categorías. Para el grupo de 18 a 25 años, los hombres que trabajan (36.3\%) o que no estudian y no trabajan $(32.7 \%)$ tienen un consumo mayor que las mujeres que se encuentran en estos grupos. 


\section{Consumo de más de 100 cigarros}

Los hombres de 12 a 17 años que trabajan (12.2\%) presentan un consumo mayor comparado con los que estudian o con los que estudian y trabajan. Aquellos que no estudian y no trabajan (9.2\%) también muestran un consumo más alto en comparación con los que estudian. En hombres de 18 a 25 años, los que estudian (13.4\%) muestran un uso menor de tabaco que quienes sólo trabajan. Por grupo de edad, los de 18 a 25 años reportan porcentajes mayores en el consumo en cada una de las categorías, que los adolescentes de 12 a 17 años.

En las mujeres, aquellas de 12 a 17 años que estudian $(0.2 \%)$ refieren un consumo menor que las que mencionaron trabajar; mientras que en aquellas de 18 a 25 años, el consumo es similar entre las distintas categorías. Por grupos de edad, aquellas de 18 a 25 años que estudian (10.4\%) o trabajan (10.7\%) presentan un uso mayor de la sustancia, en comparación con las de 12 a 17 años de las mismas categorías. Para las mujeres que no estudian y no trabajan, el consumo en aquellas de 12 a 17 años es de 0\%, mientras que en las de 18 a 25 años, se presenta un consumo del 10.6\%.

Así, los hombres de 12 a 17 años que no estudian y no trabajan presentan un consumo cercano a 10\%, mientras que en las mujeres del mismo grupo de edad, el uso de la sustancia es nulo. En el resto de las categorías, el consumo de tabaco en este grupo de edad es similar entre ambos sexos. Para aquellos de 18 a 25 años, el uso más alto se observa en los hombres que sólo trabajan (31.3\%) o en los que estudian y trabajan (22.5\%), en comparación con las mujeres de las mismas categorías.

\section{Consumo diario de tabaco}

En los hombres de 12 a 17 años y de 18 a 25 años, el uso de tabaco es similar entre las distintas categorías.

En mujeres, el consumo diario presenta porcentajes bajos en las distintas categorías y es similar en los diversos grupos.

Finalmente, en el consumo entre hombres y mujeres se observa un mayor uso en los hombres de 18 a 25 años

Tabla 2

Consumo de tabaco en población de 12 a 25 años según sexo y edad

\begin{tabular}{|c|c|c|c|c|c|c|c|c|}
\hline \multirow{2}{*}{ Sexo / Edad } & \multicolumn{2}{|r|}{ Estudia } & \multicolumn{2}{|r|}{ Trabaja } & \multicolumn{2}{|c|}{ Estudia y trabaja } & \multicolumn{2}{|c|}{ No estudia y no trabaja } \\
\hline & $\%$ & IC $95 \%$ & $\%$ & IC $95 \%$ & $\%$ & IC 95\% & $\%$ & IC $95 \%$ \\
\hline \multicolumn{9}{|c|}{$\begin{array}{l}\text { Consumo alguna vez } \\
\text { Hombres }\end{array}$} \\
\hline 12 a 17 & 21.6 & $18.33-24.87$ & 49.3 & $40.01-58.68$ & 31.5 & $22.79-40.26$ & 34.6 & $23.00-46.13$ \\
\hline 18 a 25 & 43.4 & $33.64-53.24$ & 71.3 & $66.82-75.74$ & 67.1 & $56.48-77.68$ & 73.1 & $62.18-84.00$ \\
\hline \multicolumn{9}{|l|}{ Mujeres } \\
\hline 12 a 17 & 13.3 & $10.96-15.65$ & 26.5 & 19.39-33.56 & 38.7 & $19.37-58.00$ & 25.6 & $13.99-37.28$ \\
\hline 18 a 25 & 44.6 & $34.31-54.80$ & 39.3 & $34.42-44.28$ & 52.8 & $36.85-68.71$ & 45.5 & $32.67-58.33$ \\
\hline \multicolumn{9}{|c|}{$\begin{array}{l}\text { Consumo último año } \\
\text { Hombres }\end{array}$} \\
\hline 12 a 17 & 11.3 & $8.70-14.00$ & 36.0 & $27.13-44.79$ & 19.2 & $11.71-26.63$ & 24.9 & $14.54-35.25$ \\
\hline 18 a 25 & 25.7 & $16.97-34.44$ & 47.3 & $41.99-52.56$ & 34.3 & $22.72-45.91$ & 38.6 & $26.24-50.89$ \\
\hline \multicolumn{9}{|l|}{ Mujeres } \\
\hline 12 a 17 & 6.7 & $5.07-8.28$ & 12.7 & $6.98-18.38$ & 12.9 & $3.82-22.01$ & 12.8 & $4.62-20.94$ \\
\hline 18 a 25 & 22.7 & $13.78-31.54$ & 16.4 & $12.99-19.88$ & 17.3 & $5.25-29.29$ & 14.0 & $5.87-22.078$ \\
\hline \multicolumn{9}{|c|}{$\begin{array}{l}\text { Consumo último mes } \\
\text { Hombres }\end{array}$} \\
\hline 12 a 17 & 6.4 & $4.12-8.60$ & 29.4 & 20.68-38.20 & 8.0 & $2.88-13.13$ & 18.3 & $10.12-26.43$ \\
\hline 18 a 25 & 15.6 & 8.26-23.01 & 36.3 & $31.42-41.13$ & 21.2 & $10.88-31.58$ & 32.7 & $21.34-44.08$ \\
\hline \multicolumn{9}{|l|}{ Mujeres } \\
\hline 12 a 17 & 2.1 & $1.20-2.90$ & 8.0 & $3.31-12.71$ & 4.9 & $0.00-10.34$ & 3.7 & $0.00-7.82$ \\
\hline 18 a 25 & 14.7 & $7.10-22.34$ & 12.2 & $9.01-15.39$ & 13.7 & $2.20-25.16$ & 10.9 & $3.44-18.44$ \\
\hline \multicolumn{9}{|c|}{$\begin{array}{l}\text { Consumo de } 100 \text { cigarros o más } \\
\text { Hombres }\end{array}$} \\
\hline 12 a 17 & 1.6 & $0.54-2.76$ & 12.2 & $6.25-18.24$ & 2.5 & 0.00-5.12 & 9.2 & $3.69-14.69$ \\
\hline 18 a 25 & 13.4 & $6.70-20.20$ & 31.3 & 26.57-35.93 & 22.5 & 12.98-31.94 & 28.2 & $16.86-39.45$ \\
\hline \multicolumn{9}{|l|}{ Mujeres } \\
\hline 12 a 17 & 0.2 & $0.03-0.44$ & 5.9 & 1.83-9.89 & 4.4 & $0.00-9.53$ & -- & --- \\
\hline 18 a 25 & 10.4 & $3.85-16.97$ & 10.7 & $7.73-13.72$ & 4.5 & $0.00-9.34$ & 10.6 & $3.37-17.83$ \\
\hline \multirow{2}{*}{\multicolumn{9}{|c|}{$\begin{array}{l}\text { Consumo diario } \\
\text { Hombres }\end{array}$}} \\
\hline & & & & & & & & \\
\hline 12 a 17 & 1.1 & $0.09-2.07$ & 4.8 & $1.66-8.03$ & 1.1 & $0.00-2.80$ & 5.3 & $1.02-9.67$ \\
\hline 18 a 25 & 6.7 & $1.28-12.10$ & 10.7 & $7.84-13.62$ & 2.1 & $0.00-4.26$ & 8.5 & $2.39-14.61$ \\
\hline \multicolumn{9}{|l|}{ Mujeres } \\
\hline 12 a 17 & 0.2 & $0.00-0.51$ & 1.0 & $0.00-2.17$ & -- & --- & 0.4 & $0.00-1.11$ \\
\hline 18 a 25 & 1.6 & $0.00-3.33$ & 3.7 & $1.73-5.59$ & 4.5 & $0.00-9.34$ & 2.6 & $0.00-6.37$ \\
\hline
\end{tabular}


que trabajan (10.7\%). Para el resto de los grupos, el uso diario es similar entre ambos sexos.

\section{DISCUSIÓN Y CONCLUSIONES}

El objetivo de este trabajo fue conocer la prevalencia de consumo de tabaco en los jóvenes de 12 a 25 años, según su estatus laboral o escolar; los resultados muestran que el grupo de interés, aquellos que no estudian y no trabajan, presenta un consumo mayor de tabaco en los diferentes indicadores de consumo, en comparación con quienes únicamente estudian. Esta situación también se observa en los que sólo se dedican a trabajar.

Los jóvenes que no estudian y que no trabajan tienen un riesgo mayor no sólo de iniciarse en el uso de sustancias, sino también de presentar otros problemas de salud mental, en comparación con quienes se dedican únicamente a estudiar (Benjet et al., 2012). Esto puede verse mediado por la percepción de falta de oportunidades para encontrar un trabajo sólido, las carencias económicas en el entorno familiar o el rechazo escolar ante las carreras de preferencia, con lo que se facilita la aparición de sentimientos de desesperanza, insatisfacción y problemas en la construcción de su identidad y estima (Gutiérrez et al., 2014 ; Hernández \& Benjet, 2012).

El hecho de que los jóvenes que no estudian y no trabajan tengan un porcentaje de consumo de tabaco mayor que quienes estudian -efecto observado principalmente en los hombres de 12 a 17 años- puede sugerir que la condición de no estudiar y no trabajar genera un mayor impacto en la población masculina, en la cual la necesidad de ocupar el tiempo libre, aunada a una supervisión laxa, puede facilitar el consumo de alguna sustancia (Gutiérrez et al., 2014).

Asimismo, el que los adolescentes y los jóvenes que se dedican únicamente a trabajar muestren un consumo mayor que quienes sólo estudian o estudian y trabajan, sugiere que la escuela puede representar un factor de protección ante el uso de tabaco. Estudios realizados en países en desarrollo reportan que trabajar durante pocas horas puede ser benéfico para los adolescentes, mientras que un empleo con una mayor cantidad de tiempo laboral puede asociarse con un bajo desempeño escolar y el uso de drogas, pues proporciona a los jóvenes la posibilidad de tener contacto con personas de mayor edad que ellos, así como de obtener recursos económicos para comprar las sustancias (Paschall, Flewelling, \& Rusell, 2004; Wu, Schelenger, $\&$ Galvin, 2003). A este respecto, Benjet y colaboradores (2012) mencionan que los adolescentes de 12 a 17 años que no estudian y no trabajan presentan ma- yor riesgo de fumar que los que estudian; este riesgo es aún mayor cuando los adolescentes sólo se dedican a trabajar.

Este fenómeno no se da únicamente en adolescentes que sólo trabajan, sino también en aquellos que estudian y trabajan. Investigaciones realizadas en población escolar reportan un incremento en el uso de tabaco entre quienes laboran cuatro horas diarias, comparados con los que se dedican exclusivamente a estudiar; el consumo presenta un mayor porcentaje cuando la jornada laboral es de ocho horas diarias (Chávez et al., 2013; Villatoro et al., 2014).

Los resultados del presente trabajo también dan cuenta de que a mayor edad, existe un consumo superior de tabaco en las diferentes categorías analizadas, principalmente entre quienes sólo estudian o sólo trabajan. Este incremento del consumo con la edad puede estar mediado por la tolerancia social que perciben los adolescentes ante el uso de cigarros y con la consecuente disminución en la percepción de riesgo (Villatoro et al., 2014); al ser mayores, tienen más disponibilidad y acceso a los cigarros, así como una disminución de la supervisión y el monitoreo de los padres (Mrug, Gaines, Su, \& Windle, 2010; Palacios \& Andrade, 2008; Tilton \& Galambos, 2003).

Si bien en nuestro país la prevalencia del consumo de tabaco en el último año en la población general no es tan alta como la que se presenta en otros países de América, cuyos porcentajes van de $38.2 \%$ en Chile a $7.7 \%$ en El Salvador (OEA \& CICAD, 2015), es importante reforzar y ampliar las acciones preventivas y de intervención que se han realizado desde la firma y ratificación del Convenio Marco para el Control de Tabaco de la OMS. Como se ve reflejado en este trabajo, los jóvenes de 12 a 25 años que tienen pocas oportunidades de continuar sus estudios y que se insertan en el mundo laboral a una edad temprana, o bien, que no reciben oportunidades ni de estudio ni de trabajo, presentan mayores probabilidades de convertirse en fumadores regulares, lo cual, con el tiempo, puede llevar al consumo problemático o a la dependencia de la sustancia.

Aun cuando la realización de acciones oportunas en este sector de la población es fundamental para prevenir o disminuir el consumo de tabaco, también resulta relevante dotar a los adolescentes y jóvenes de recursos personales que les permitan afrontar las situaciones a las que se ven expuestos, como la presión por parte de amigos, familiares o personas externas para que consuman sustancias; asimismo, es indispensable ofrecerles alternativas que potencialicen y sustenten su toma de decisiones, así como garantizar que cuenten con espacios saludables de ocio y tiempo libre. Lo anterior encaminado a mantener una 
buena salud mental en la población joven, en ocasiones la más vulnerable.

\section{FUENTES DE FINANCIAMIENTO}

La Encuesta Nacional de Adicciones 2011 fue financiada por la Secretaría de Salud y la Comisión Nacional contra las Adicciones. Para la elaboración de este manuscrito no se recibió ningún financiamiento adicional.

\section{CONFLICTOS DE INTERÉS}

Los autores de este artículo declaramos que no existe ningún conflicto de interés en la realización de este manuscrito.

\section{AGRADECIMIENTOS}

Agradecemos a la Comisión Nacional contra las Adicciones por el apoyo brindado en la realización del estudio que permite presentar el actual trabajo. La encuesta se hizo en coordinación con el Instituto Nacional de Salud Pública.

\section{REFERENCIAS}

Anderson, P. (2006). Global use of alcohol, drugs and tobacco. Drug and Alcohol Review, 25(6), 489-502.

Benjet, C. (2009). La salud mental de la niñez y la adolescencia en América Latina y el Caribe. En Pan American Health Organization (PAHO) (Ed.), Epidemiología de los trastornos mentales en América Latina y el Caribe. Publicación Científica y Técnica, 632, 234-242.

Benjet, C., Hernández-Montoya, D., Borges, G., Méndez, E., Medina-Mora, M. E., \& Aguilar-Gaxiola, S. (2012). Youth who neither study nor work: Mental health, education and employment. Salud Pública de México, 54(4), 410-417.

Chávez, J., Villatoro, J., Robles, L., Bustos, M., Moreno, M., Oliva, N., Fregoso, D.,... Paredes, A. (2013). Encuesta escolar sobre adicciones en el Estado de Jalisco 2012. México, D.F.: Consejo Estatal contra las Adicciones de Jalisco, Instituto Nacional de Psiquiatría Ramón de la Fuente Muñiz.

D’Alesandre, V. (2010). Adolescentes que no estudian ni trabajan. Cuaderno 04, International Institute for Educational Planning, IIPE-UNESCO, Organización de Estados Iberoamericanos para la Educación, la Ciencia y la Cultura (OEI). Recuperado en http://www.siteal.iipe-oei.org.

Department of Health and Human Services-USA, Center for Disease Control and Prevention, Organización Mundial de la Salud. (2011). Preguntas sobre tabaco destinadas a encuestas. Serie de preguntas básicas de la Encuesta Mundial de Tabaquismo en Adultos (GATS) (2a Edición). Atlanta: Author.
Gutiérrez, R., Martínez, K., Pacheco, A., \& Benjet, C. (2014). La construcción social de la identidad en los jóvenes que no estudian ni trabajan. Revista Iberoamericana de Ciencias, 1(7) 1-12.

Hernández, D. S., \& Benjet, C. (2012). Los ninis como problema emergente para la salud pública. Revista Mexicana de Pediatría, 79(1), 40-45.

Instituto Nacional de Estadística y Geografía. (2012). Encuesta Nacional de Ingresos y Gastos de los Hogares 2012 (ENIGH). Recuperado de http://www.inegi.org.mx/est/contenidos/proyectos/encuestas/hogares/regulares/enigh/enigh2012/tradicional/ default.aspx

Instituto Nacional de Psiquiatría Ramón de la Fuente Muñiz, Instituto Nacional de Salud Pública, Secretaría de Salud (2012). Encuesta Nacional de Adicciones 2011: Reporte de Tabaco. Reynales-Shigematsu, L. M., Guerrero-López, C. M., Lazcano-Ponce, E., Villatoro-Velázquez, J. A., Medina-Mora, M. E., Fleiz-Bautista, C., Téllez-Rojo, M.M., Mendoza-Alvarado, L.R., Romero-Martínez, M., Gutiérrez-Reyes, J.P., Castro-Tinoco, M., Hernández-Ávila, M., Tena-Tamayo, C., Alvear-Sevilla, C. \& Guisa-Cruz, V. México DF, México: INPRFM; 2012. Disponible en www.inprf.gob.mx, www.conadic.gob.mx, www.cenadic.salud. gob.mx, www.insp.mx

Johnston, L. D., O’Malley, P. M., Miech, R. A., Bachman, J. G., \& Schulenberg, J. E. (2015). Monitoring the Future national results on drug use: 1975-2014: Overview, key findings on adolescent drug use. Ann Arbor: Institute for Social Research, The University of Michigan.

Mrug, S., Gaines, J., Su, W., \& Windle, M. (2010). School-level substance use: effects on early adolescents' alcohol, tobacco, and marijuana use. Journal of Studies on Alcohol and Drugs, 71(4), 488-495.

National Center on Addiction and Substance Abuse (CASA) at Columbia University \& Califano, J. A. (2007). Wasting the best and the brightest: substance abuse at america's colleges and universities. New York: CASA.

Nuño-Gutiérrez, B.L., Álvarez-Nemegyei, J., Madrigal-De León, E., \& Rasmussen, B. (2005). Prevalencia y factores asociados al consumo del tabaco en adolescentes de una preparatoria de Guadalajara, Jalisco, México. Salud Mental, 28(5), pp. 64-70.

Nuño-Gutiérrez, B.L., Álvarez-Nemegyei, J., Madrigal-De León, E., \&Tapia-Curiel, A. (2008). Factores asociados a los patrones de consumo de tabaco en adolescentes escolares. Revista Médica del Instituto Mexicano del Seguro Social, 46(1), 19-26.

Organización de los Estados Americanos, Comisión Interamericana para el Control del Abuso de Drogas. (2015). Informe sobre uso de drogas en las Américas 2015. Recuperado de http://www. cicad.oas.org/apps/Document.aspx?ld=3209

Organization of American States, Inter-American Drug Abuse control Commission. (2010). Comparative analysis of student drug use in Caribbean countries. Recuperado de http://www.cicad.oas. org/Main/pubs/StudentDrugUse-Caribbean2011.pdf

Organización Panamericana de la Salud, Instituto Nacional de Salud Pública. (2010). Encuesta Global de Tabaquismo en Adultos. México 2009. México: Autor. 
Palacios, J.R., \& Andrade, P. (2008). Influencia de las prácticas parentales en las conductas problema en adolescentes. Investigación Universitaria Multidisciplinaria, 7,7-18.

Paschall, M. J., Flewelling, R. L., \& Rusell, T. (2004). Why is work intensity associated with heavy alcohol use among adolescents? Journal of Adolescent Health, 34, 79-87.

Pérez, B., Rivera, L., Atienzo, E., De Castro, F., Leyva, A., \& Chávez, R. (2010). Prevalencia y factores asociados a la ideación e intento suicida en adolescentes de educación media superior de la República mexicana. Salud Pública de México, 52, 324-333.

Reddy-Javobs, C., Téllez-Rojo, M.M., Meneses-González, F., Campuzano-Rincón, J., \& Hernández-Ávila, M. (2006). Pobreza, jóvenes y consumo de tabaco en México. Salud Pública de México, 48(1), S83-S90.

Reynales-Shigematsu, L. M., Rodríguez-Bolaños, R., Ortega-Ceballos, P., Flores-Escartín, M. G., Lazcano-Ponce, E., \& Hernández-Ávila, M. (2011). Encuesta de Tabaquismo en Jóvenes. México: Instituto Nacional de Salud Pública.

Salazar, J., Torres, T., Reynaldos, C., Figueroa, N., \& Araiza, A. (2011). Factores asociados a la delincuencia en Guadalajara, Jalisco. Papeles de Población, 17(68), 103-126.

Székely, M. (2011). Jóvenes que no estudian ni trabajan: un riesgo para la cohesión social en América Latina. Santiago de Chile: CIEPLAN.
Tilton, L. C., \& Galambos, N. L. (2003). Adolescents' characteristics and parents' beliefs as predictors of parents peer management behaviors. Journal of Research on Adolescence, 13(3), 269-300.

Villalobos, A., \& Rojas, R. (2007). Consumo de tabaco en México. Resultados de las Encuestas Nacionales de Salud 2000 y 2006. Salud Pública de México, 49(2), S147-S154.

Villatoro, J., Mendoza, M.A., Moreno, M., Oliva, N., Fregoso, D., Bustos, M.,... Medina-Mora, M.E. (2014). Tendencias del uso de drogas en la Ciudad de México: Encuesta de estudiantes, octubre 2012. Salud Mental, 37(5), 423-435.

Villatoro, J.A., Sandoval, A.I., Moreno, M., López, M.A., Gutiérrez, M., \& Magaña, E.N. (2010). Drug, Alcohol and tobacco consumption associated factors in high school and college students. El Faro la voz de la red, 3(3). Disponible en http:// www.nhsn.med.miami. edu/x806.xml

Welte, J., Barnes, G., Tidwell, M. C., \& Hoffman, J. (2011). Tobacco use, heavy use, and dependence among adolescents and young adults in the United States. Substance Use \& Misuse, 46 , 1090-1098.

Wu, L., Schelenger, W., \& Galvin, D. (2003). The relationship between employment and substance use among students aged 12 to 17 . Journal of Adolescent Health, 32, 5-15. 